\title{
A Parallel Processing Framework for Real-Time Software-Only Video Compression for Broadcast and Mobile Services
}

\author{
Lizhi Zheng and John Cosmas \\ School of Engineering and Design, Brunel University \\ Uxbridge, Middlesex, UB8 3PH, U.K.
}

\begin{abstract}
This paper develops a generic parallel processing framework on embedded multicomputer systems for real-time video compression for convergent broadcast and cellular services. This framework allows easy prototyping of new parallel video compression algorithms and integration with video applications. Using the proposed parallel processing framework, a parallel H.263 encoder has been implemented on a PowerPC processor-based Mercury RACE multicomputer. The experimental results show promising parallel speedups. Real-time performance was achieved for QCIF video using the non-optimized reference software. The parallel processing framework not only can be configured with different number of processors on an embedded multicomputer system but also is portable onto varied embedded multicomputer platforms. Therefore, this paper provides a software implementation solution as an alternative to hardware video compression for broadcast and mobile services.
\end{abstract}

\section{KEYWORDS}

broadcast and mobile service, parallel processing framework, real-time video compression

Reprint requests to: Lizhi Zheng, School of Engineering and Design, Brunel University, Uxbridge, Middlesex UB8 3PH, U.K.; e-mail: Lizhi.Zheng@brunel.ac.uk 


\section{INTRODUCTION}

Facing the challenge of converging mobile and broadcast services and networks, this paper proposes a generic parallel framework to achieve realtime software video compression at the service provisioning side. Broadcast and mobile services and networks' convergence is very promising, which will bring great benefits to end-users. On the one hand, today's mobile communication networks can offer mobility and interactivity to the end-users and the possibility to receive information on the users' personal interests. The available mobile services, however, evolving from GSM (Global System for Mobile Communications), WAP (Wireless Application Protocol Technology), GPRS (General Packet Radio Service) to UMTS (Universal Mobile Telephony System) technology, cannot provide a sufficient bit rate at a reasonable cost to provide rich multimedia services to a large audience. On the other hand, digital technology is the latest progress in the evolution of television. In today's television industry, the transition from analogue to digital broadcasting is the main topic of interest, where digital television can dramatically improve television transmission quality and increase the number of public television stations. The DVB (Digital Video Broadcasting) channel is a unidirectional broadband channel carrying video, audio, and data from the broadcast service provider to the end-users. Digital video broadcasting provides broadcast services at greater than $2 \mathrm{Mbps}$ and is a cost efficient multicast system with the ability to broadcast rich multimedia content to a large audience. Nevertheless, DVB does not provide a built-in return channel, which is essential to enable interactive TV and data services.

Therefore, with broadcast and mobile services and networks being complementary to each other, promising interactive services including different kinds of multimedia content (e.g. audio-visual, image, internet page, text) can be prepared and delivered to end-users via broadcast or mobile networks. A typical architecture for a converged DVB and mobile telecom system consists of Service Provisioning System, Broadcast and Mobile Access Network, and Terminals, as shown in Fig. 1.

The Service Provisioning System produces the content (which includes video encoding), creates and announces the converged services. The Broadcast and Mobile Access Network decides over which network this media is 


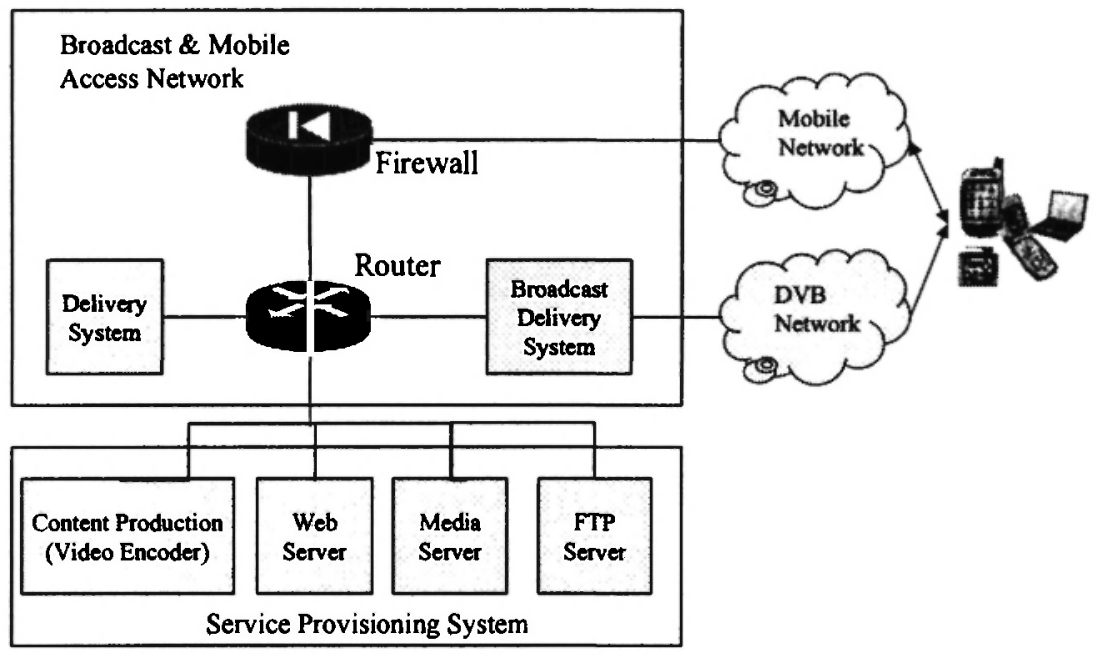

Fig. 1: Basic architecture for a converged DVB and mobile system

depending on the demand for services from the end-users. Mobile terminals could be of many types with different capabilities (e.g. screen size, processing power, and battery life etc.). They can be Tablet PC, PDA (Personal Data Assistant), or mobile phone, and others.

When demand is high, the video encoder is required to deliver high bitrate (e.g. $>256 \mathrm{Kbps}$ ) video with higher resolution on the IP over the DVB network. When demand is low, the Service Provisioning System is required to deliver low bitrate (e.g. $<128 \mathrm{Kbps}$ ) video with lower resolution on the mobile network. Hence, different qualities of video clips having different resolutions (e.g. QCIF, CIF, or CCIR601, etc.) must be prepared for realizing multimedia interactive data services for end-users. To realize live television broadcast and interactive services with full control of the video, encoding digital video signal in real-time is critical. "Real-time" performance is typically considered to be $25 / 30$ frames per second. Real-time encoder transforms live video into compressed bitstream, allowing a viewer to access the digital video stream just seconds after the live event took place. For the televisionprogram-related content, the raw video content must be encoded fast enough to be ready for broadcast with the main television program or for streaming/ downloading via mobile network. 
The challenge is that real-time video compression demands a large amount of computing power, particularly with the introduction of highly computational, expensive encoding algorithms such as H.264 (ITU-T, 2003; Wiegand et al., 2003). Traditionally, compression is achieved by using special-purpose hardware platforms, such as Harmonic's DiviCom MV encoder (Harmonic, 2004), Envivio's 4Caster encoder (Envivio, 2004), and Optibase's MPEG MovieMaker encoder (Optibase, 2004). Hardware implementation, however, provides limited flexibility and cannot be modified for later extensions. The architecture design usually requires a regular control paradigm and data flow of the algorithms that may not be suitable for most contentbased techniques. Above all, such devices are expensive and usually take a relatively long time to design. An alternative is to provide a software implementation solution using low-cost, high-performance parallel systems, but the difficulty is that the utilization of all the available computational power in such systems appears to require a tremendous programming effort on the part of developers.

This paper suggests a suitable software framework to enable the effective use of emerging embedded multicomputer systems to achieve real-time software video compression. The rest of the paper is organized as follows. Section 2 discusses different implementation approaches used in software video compression in relation to this work. Section 3 introduces the proposed parallel processing framework. Section 4 discusses the implementation of a parallel H.263 encoder using this framework on a PowerPC processor-based Mercury RACE multicomputer and the experimental results. Section 5 summarizes the contributions of this research to the future developments.

\section{RELATED WORK}

The software implementation approaches for video compression can be broadly classified into four categories according to their implementation platforms-massive parallel systems, shared memory parallel systems, multiprocessor DSP systems, and cluster of computers.

Massive parallel systems such as Intel Touchstone Delta, Intel Paragon, Cray T3D, and IBM SP2 have been used for software video compression. A 
parallel MPEG-1 video encoder (MPEG-1 video group. 1993) implemented on the Intel Touchstone Delta and the Intel Paragon was described in Shen \& Delp (1995). Although faster-than-real-time performance has been claimed, one drawback is that the complete video sequence must be available before encoding begins. This limitation is impractical for real-time encoding applications. A fine-grained software-only MPEG-2 video encoder (MPEG-2 video group, 1995) on Intel Paragon XP/S and its performance was described in Akramullah et al. (1995). The MPEG-2 achieved a maximum frame rate of 31.14 frames per second (fps) using 330 processors. A visualization video compression application implemented on massively parallel computer CRAY T3D was described in Nicolas and Jorda (1996). Depending upon the complexity of the compression algorithm, 8-12 images were compressed, decompressed and visualized per second. An H.261 encoder (ITU-T, 1990) was implemented using a data-parallel approach on the IBM SP2 with 24 processors (Yung \& Chu, 1998). Although these massive parallel systems often provide real-time performance on large number of processors, their system efficiency is relatively low (range from $32 \%$ to $40 \%$ ). Moreover, these parallel systems, normally used for science computing, are very expensive and not commercially viable. Not many users can have exclusive access to these systems.

Parallel encoders have also been implemented on shared-memory parallel systems. Barbosa et al. (1999) proposed and implemented a simple sharedmemory parallel algorithm for MPEG-1 video encoding based on the parallel execution of different coarse-grained tasks on the Sun enterprise 100000 containing 32 processors. The Group of Pictures (GOP) and frame encoding tasks were mapped to the parallel system using a multithreaded methodology. In Barbosa et al. (1999), an MPEG-2 encoder was implemented using a Sun multiprocessor with 32 processors. An H.264 encoder using multithreading techniques was implemented on multiprocessor Intel PCs including with Hyper-Threading technology ( $\mathrm{Li} \&$ Chen, 2004). A shared memory system, IBM Power Visualization System with 32 Intel i860 processors and 1 GB shared memory, was used for a parallel implementation of an MPEG-2 compliant, a constant bit rate video encoder (Tiwari \& Viscito, 1996). The main problem of shared memory systems is that a bottleneck occurs when a number of processors attempt to access the global memory store at the same 
time. This drawback not only limits the communications but also destroys determinism within the operations. Shared memory is limited by this bottleneck for scaling within multiprocessor systems.

Multiprocessor DSP systems are used for video compression as well. A parallel implementation of H.261 encoder on a TMS320C80 MVP was reported in Leung et al. (2000). The performance of $30.7 \mathrm{fps}$ was achieved on 4 processors for QCIF. A parallel implementation of $\mathrm{H.263}$ video CODEC on a TMS320C80 MVP was reported in Lin et al. (1997). The system achieved QCIF encoding at $7.5 \mathrm{fps}$ after reducing the computation complexity of the encoder. A parallel implementation of the H.263 video encoder using four SHARC (ADSP-21062) DSPs was presented for video conferencing applications (Kolinummi et al., 2000). Using the optimized motion-estimation algorithms and assembly coding, real-time encoding was achieved with a QCIF sized picture. Multiprocessor DSP systems offer a performance typical of parallel machines together with limited cost. Memory size and bandwidth are the limiting factors, however. With limited fast internal memory and limited bandwidth to external memory, a bottleneck often appears between the processor and the data. The DSP processors use a high-speed on chip RAM to accelerate processing, but the programmer must manage the staging of instructions and data explicitly. Thus, multiprocessor DSP systems require a very careful tuning of code and very good compilers to put all their power to work. Furthermore, multiprocessor DSP systems have limited expandability and flexibility.

The Cluster of Workstations used for software video encoding has also been reported (Akramullah et al., 1997; Fernández \& Malumbres, 2002; He et al., 1999). Compared to massive parallel systems, implementations on a cluster of workstations achieve better efficiency on a small number of processors, but the actual frame rate is usually lower than real-time performance. Their inherent inefficient inter-processor communication can be a problem. Moreover, like MPP systems, a cluster of workstations is difficult to integrate into other multimedia platforms owing to the constraints of physical size and power.

Real-time software video compression not only requires huge amount of computation but also demands a balanced architecture of flexible $\mathrm{I} / \mathrm{O}$ and memory bandwidth. The overall system performance for software real-time 
video compression requires many processors communicating over high bandwidth links to process a continuous flow of video data that are captured from video camera, storage, or other devices. Real-time software video compression also has constraints of cost, power, weight, and physical size. The embedded multicomputer systems are ideal candidates for real-time video encoding because they provide scaleable high-performance processing power and a balanced architecture of flexible I/O and memory bandwidth. Because the internal bandwidth is scaleable in an embedded multicomputer system, the throughput naturally increases with the number of functional elements in the system (Einstein, 1997; Kuszmaul, 1995). This feature is a big advantage over bus systems in which internal bandwidth decreases with the number of functional elements. No other report on the implementations of software video compression on such embedded multicomputer systems has been found in the literature.

As discussed above, a number of software implementation examples of H.261 have been reported (Yung \& Chu, 1998; Leung et al., 2000), H.263 (Lin et al., 2000; Kolinummi et al., 2000; Akramullah et al., 1997), H.264 (Li \& Chen, 2004; Fernández \& Malumbres, 2002), MPEG-1 (Barbosa et al., 1999; Shen \& Delp, 1995), MPEG-2 (Akramullah, et al., 1995; Barbosa et al., 1999), and MPEG-4 (MPEG-4 video group, 1998; He et al., 1999) on various parallel systems. All these software implementations, however, tend to focus on the parallelization methodologies of the video algorithms and reporting implementation results. Thus, the parallel-processing framework proposed in the present paper will be useful as a software platform for parallel video encoding algorithms.

\section{PARALLEL PROCESSING FRAMEWORK}

The parallel processing framework was designed using Single Program Multiple Data (SPMD) (Hatcher et al., 1991), a common approach for implementing data parallel computations in Multiple Instruction Multiple Data (MIMD) environments. The computation is performed by a set of identical tasks, with each a processor being assigned a different portion of the data domain. MIMD is a Flynn's taxonomy, a widely adopted method of 
classifying parallel computer architectures, which is based on the presence of single or mutliple streams of instruction and data. MIMD architectures are made up of several independent processors that are capable of executing individual instruction streams, possibly each processor executing a different program. Most current supercomputers, networked parallel computer 'grids', and multi-processor SMP computers are typical examples of MIMD architectures. The following sections will introduce the communication topology and the architecture, mapping strategy, and synchronization of this framework.

\subsection{Communication Topology}

The design of communication typology founds the basis of message passing in the proposed Parallel Processing Framework. Figure 2 shows that the processing nodes, including host processor and compute nodes (CNs), are designed as a linear pipeline of processors. The host processor communicates with the $\mathrm{CN}$ via shared memory.

Each $\mathrm{CN}$ has a local communication buffer, and each $\mathrm{CN}$ has a pointer to its immediate neighbor's communication buffer. The entire processing pipeline, therefore, operates with commands issued from the host and acted upon by each $\mathrm{CN}$. A message propagates through the pipeline and returns to the host communication buffer. The message is designed as an inter-processor communication "command packet" structure ipcInfo. A message example including flags, action commands and computation parameters is shown in Fig. 3. In Fig. 3, message flag is used by each $\mathrm{CN}$ to indicate whether the message from the previous $\mathrm{CN}$ has been received or not. If received, then it goes into the loop and carries out the processing. Otherwise, it stays idle.

Next, operation is used for the host processor to signal which computation operation is required on each $\mathrm{CN}$, and each $\mathrm{CN}$ is acting to respond to the command received from the host processor to carry out the operation.

Then, response is used for the last $\mathrm{CN}$ to signal to the host processor, whether the processing on $\mathrm{CN}$ side has been successful or not.

Finally, processing_status is used for the each $\mathrm{CN}$ to signal to the next $\mathrm{CN}$ whether the processing has been finished or not. 


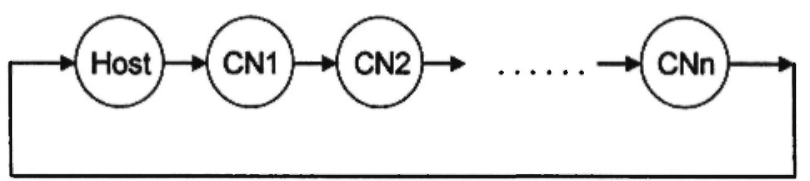

Fig. 2: Linear pipeline communication topology

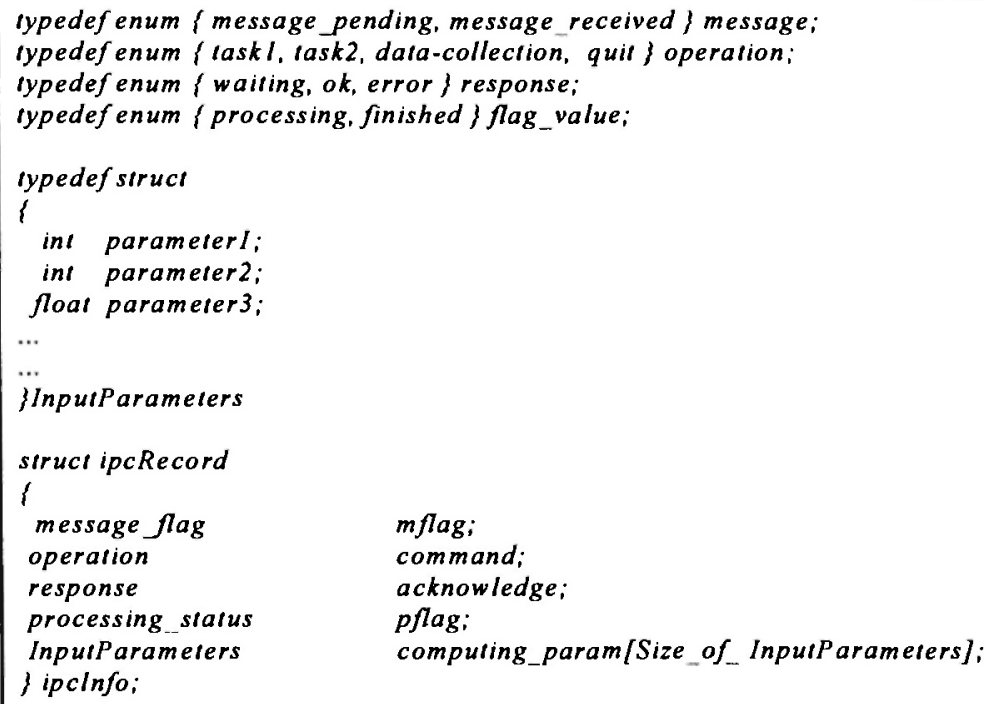

Fig. 3: Structure of a message example: ipclnfo

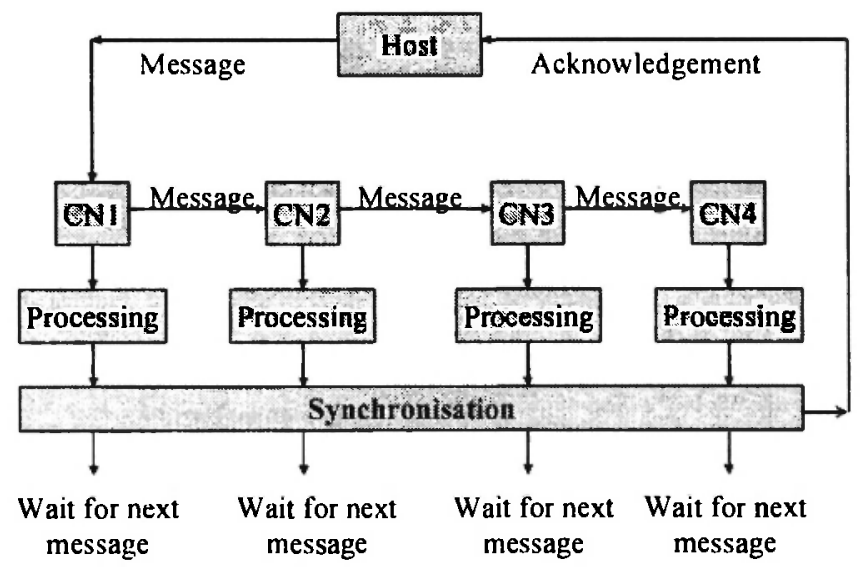

Fig. 4: Parallel Processing Framework architecture 


\subsection{Framework Architecture}

Based on the linear communication topology, Fig. 4 shows the architecture of the Parallel Processing Framework. The host processor is responsible for booting all $\mathrm{CNs}$, creating all the shared memory, starting/terminating the processing and performing $1 / O$ operations, such as reading input data, saving computing results, updating the command and computation parameters etc. The flow chart on the host processor is shown in Fig. 5.

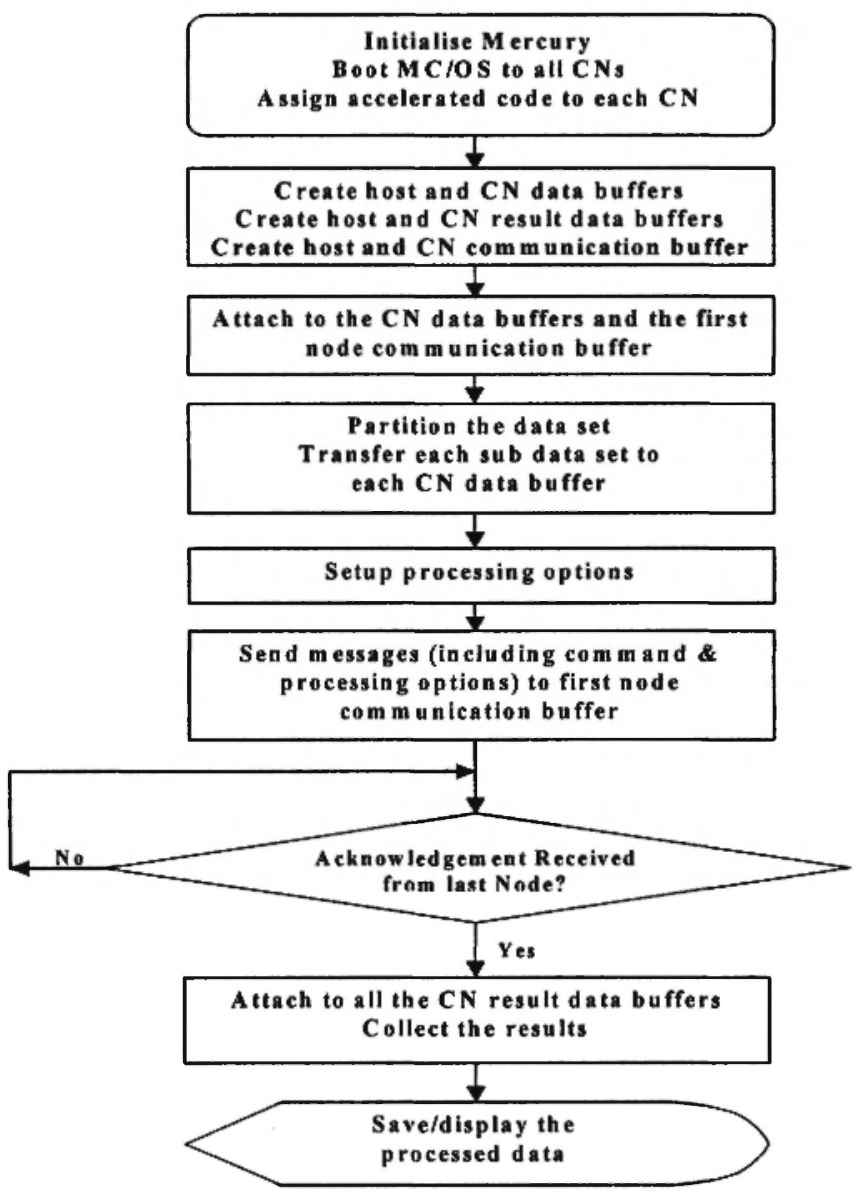

Fig. 5: Host PC flow chart 
Each node is acting as a server to respond to the commands received from the host processor to carry out the operations. After the parallel machine is initialized or finishes a task, each $\mathrm{CN}$ is idle waiting for the incoming message. At this stage, the message flag status on each $\mathrm{CN}$ is set to "message pending". When a message is received (flag status is set to "message received"), each $\mathrm{CN}$ goes into the loop and carries out the operation. When finishing the task, each $\mathrm{CN}$ sets the message status flag back to "message pending" and waits for the next incoming message.

In the computation part, it should be identified which part of the sequential code can or cannot be parallelized. We call the sequential code such as the I/O part of a program that cannot be parallelized "non-accelerated code" and the code that can be parallelized "accelerated code", respectively. The non-accelerated code is scheduled to run on the host processor. The accelerated codes are scheduled to run on the multiprocessors.

Then, we need to identify the coding parameters and data that are required for the accelerated code. Normally these coding parameters and data are packaged on a host processor and sent to the multiprocessors. Within the accelerated code, we then need to identify different computation stages and the data dependencies within each computation stage. The accelerated procedures for all the nodes are identical except the message passing part, as shown in Fig. 6.

Assuming two computation tasks in the video compression pipeline, Figure 7 shows the pipeline pseudo code for host processor and all the CNs.

\subsection{Mapping Strategy and Synchronization}

Mapping strategy. Mapping strategy is concerned with data partition that determines the best method for distributing input data across CNs of the multiprocessor system. As shown in Fig. 8, the input data are divided into sub-data equally or unequally which depends on the regularity of the input data and number of processors. Each sub-data is individually transferred to each compute node's DRAM and processed respectively.

The aim of data partition is to minimize the inter-processor communication overhead. The proposed framework does not suggest a particular 

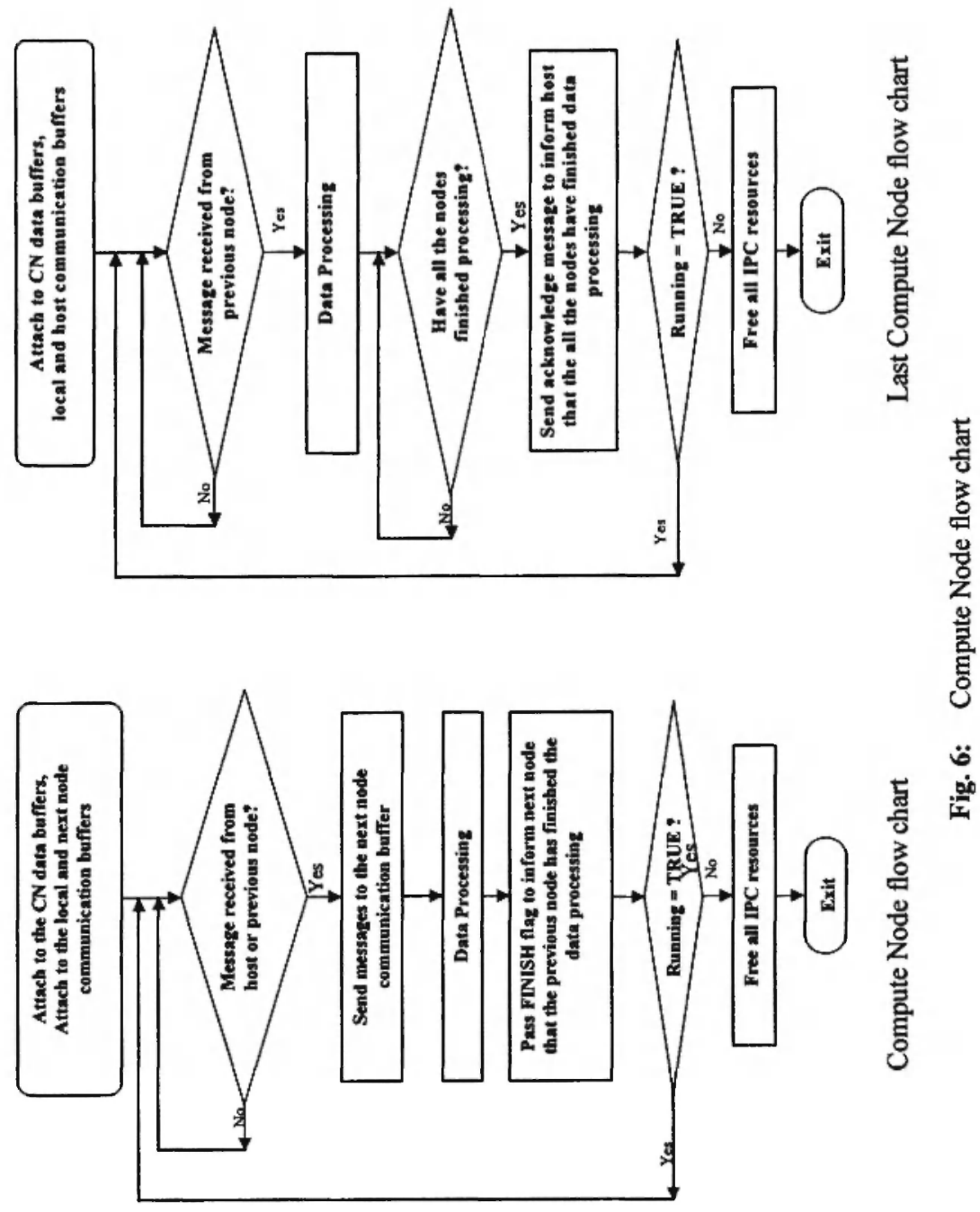

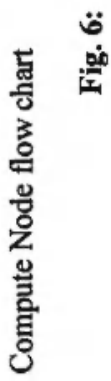


Vol. 15, No. 1-4, 2006
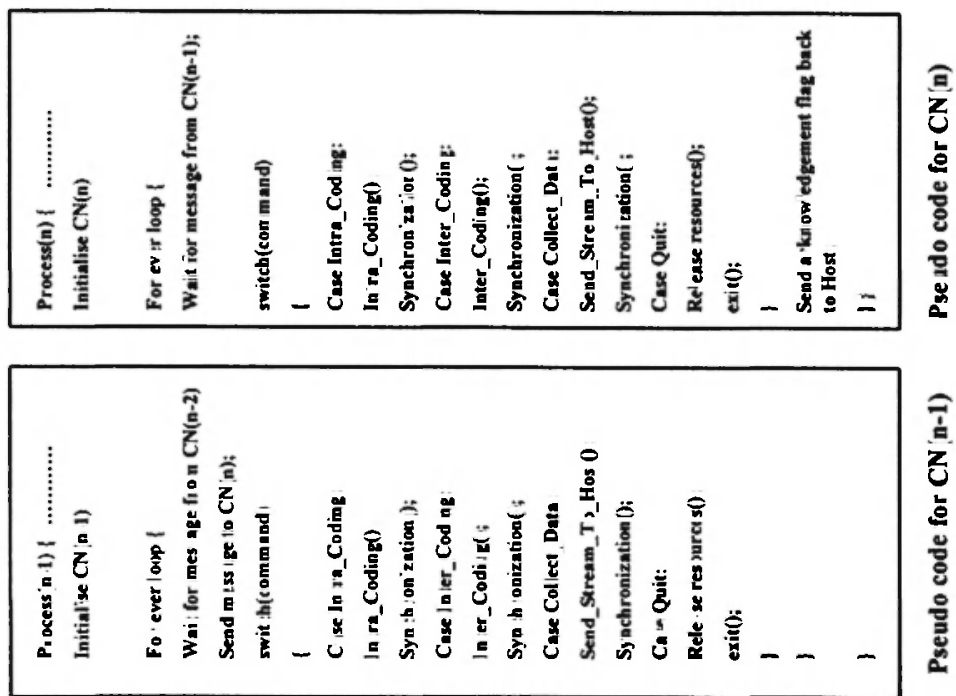

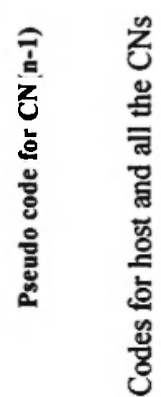

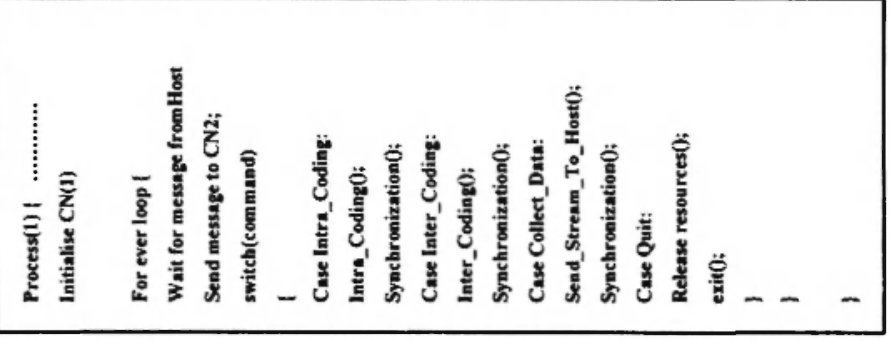

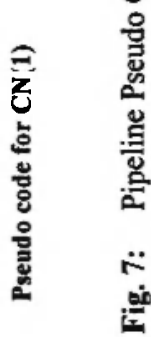

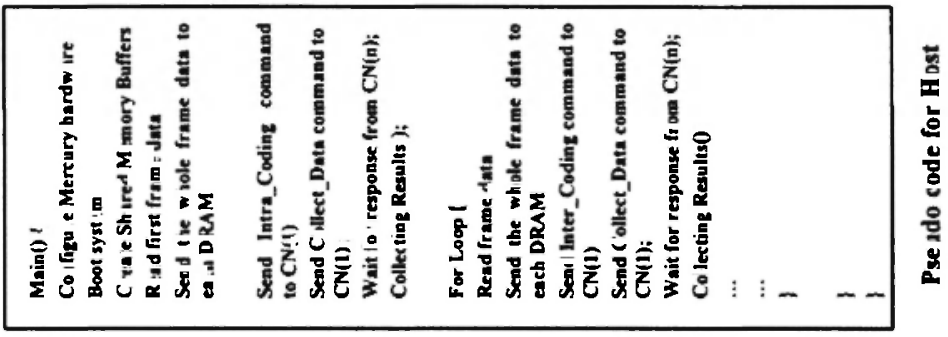




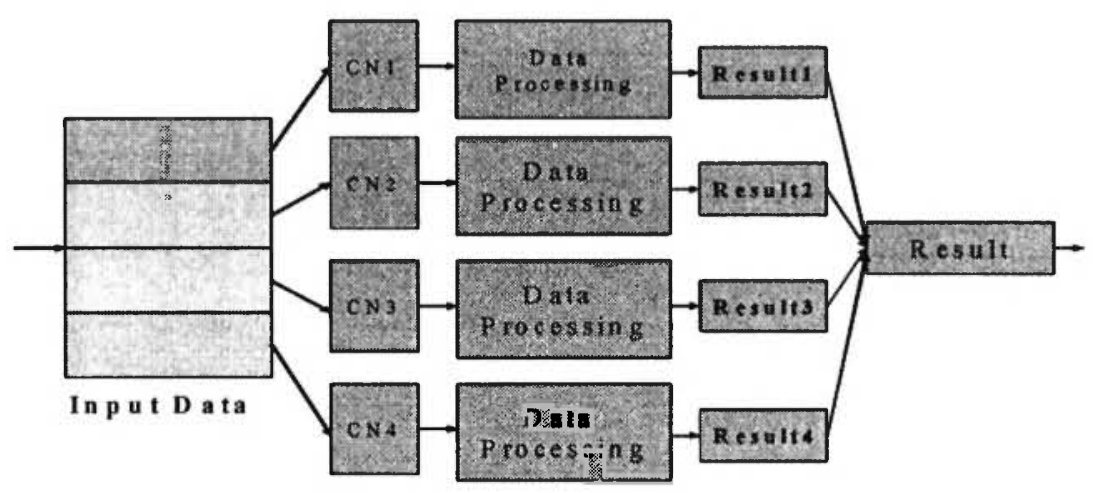

Fig. 8: Data partition for parallel processing

mapping strategy, leaving the choice to application developers to decide which mapping strategy is more suitable for their application.

Synchronization. Synchronization determines the scheduling of communications between phases of computation, including data synchronization and processing synchronization. For data synchronization, application developers must determine that the data are ready before processing. For processing synchronization, developers must make sure that the next task begins after all the processors finish the current task.

In the proposed framework, the synchronization is carried out by sending and receiving flags among the processors using the linear pipeline communication topology in Fig. 1. Application developers must make sure that the sub data is transferred to the local memory of each node and ready before sending command messages to the nodes. Under some circumstances, application developers must also make sure that the encoding results on some particular nodes are ready because another node needs to refer to these results in order to carry out further encoding operations.

After the parallel machine is initialized or finishes a task, each $\mathrm{CN}$ is idle waiting for the incoming message flag from the host processor. At this stage, the message flag status is set to message pending. When a message is received (flag status is set to message_received), each $\mathrm{CN}$ goes into the loop 
and carries out the operation. When finishing the task, each $\mathrm{CN}$ sets the message flag status back to message_pending, waiting for the next incoming message. For each operation, each node might finish at different time, so to synchronize these nodes, the first node sends a message (defined as processing status, either finished or processing) to the next node.

The whole process proceeds as follows: when the first node finishes data processing, it sends a flag to the next node, indicating that it has finished processing. After the second node has finished processing its own data, it waits until the flag has arrived from the first node. Then, the second node passes the flag to the next node and so on until the flag reaches the last node. The last node in the pipeline sends an acknowledgement signal and information of each computed partial result to the PC host, indicating that all the nodes have finished processing. Next, the host processor issues a command to tell each node to send its computed partial result back to the host processor. Finally, the host processor collates all the partial results and saves the collated result or displays it on screen.

\subsection{Data Transfer}

Data transfer is the key to message passing and synchronizing processes among multiprocessors. Data transfer is carried out using two approachesMemory Copy and DMA transfer.

3.4.1 Memory Copy. The Memory Copy is an easier way to transfer data between processors compared with DMA transfer. This method has only to specify the source buffer, destination buffer, and the size of the data to be transferred, as shown below:

memcpy (destination buffer, source buffer, size of the data)

The disadvantage of the Memory Copy technique, however, is its use of CPU time. Hence, in this framework, the Memory Copy approach is used for transferring small size of message or data.

3.4.2 Direct Memory Access transfer. The most efficient message passing method for multicomputer systems is Direct Memory Access (DMA). A transfer plan specifies how data are moved between endpoints. The transfer plan encapsulates how a transfer will occur and is created by specifying the endpoints, the engine (e.g. CPU or DMA), and optional transfer policies (e.g. fabric routing policies). Once a transfer plan is created, that plan executes 


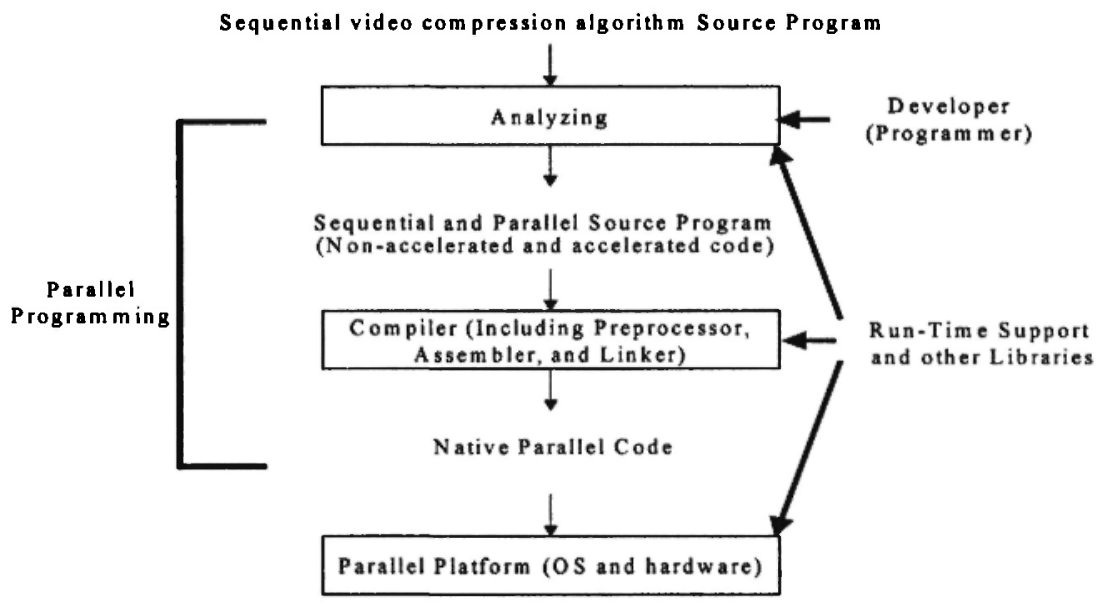

Fig 9: Components of a parallel program system

with very low latency. The transfer rate is faster than the Memory copy. Hence, in this framework, the DMA transfer approach is used for transferring bigger size of message or data.

\subsection{Parallel Program Environments}

This section shows how the proposed framework can be used by video application developers to develop parallel video compression algorithms. A typical parallel program system has a structure as shown in Fig. 9. An algorithm is first developed to solve an application problem. Then a developer (programr) implements the algorithm in a high-level language (the source program). Here, we assume that a sequential program has been written.

3.5.1 Analyzing the sequential program. Based on a sequential source program of a video codec, we first have to identify the parts with I/O functions, such as reading data from hard disk, sensor or live camera buffer, setting up coding parameters, and saving computing results. The code for $1 / O$ parts is the sequential part that cannot be parallelized. Then in the computation part, the sequential code that can or cannot be parallelized should be identified. Within the non-accelerated code, we need to identify the coding parameters and command data that are needed for the accelerated code. Normally these coding parameters and command data are packaged on the 
host processor and sent to the multiprocessors. Within the accelerated code, we then need to identify different computation stages and the data dependencies within each computation stage.

3.5.2 Writing the non-accelerated and the accelerated codes. Based on the analysis results of the sequential program, we can first design the message structure with the identified coding parameters and computation stages, following the example shown in Fig. 3. Then based on Fig. 5 and Fig. 6, we insert the supporting parallel platform's libraries into the non-accelerated code and accelerated codes. The focus is on knowing how to integrate the software libraries into the program. For example, of particular concern are how to boot up the machine, how to spawn executable codes on the multiprocessors, and particularly how to create shared memory buffers and transfer data among processors. As in multicomputer systems, a processor can access only its own memory. Memory endpoints are allocated on the host processor and all the CNs for sending and receiving messages and data, as shown in Fig. 10. The memory endpoints are called Shared Memory Buffers (SMBs).

3.5.3 Compiling. The non-accelerated code scheduled on host processor is compiled using a compiler designed for the host processor (such as Microsoft Visual $\mathrm{C}++$ compiler). A compiler provided by the parallel system vendor translates the accelerated-code into the native code to execute on a parallel platform, which includes the operating system and the underlying parallel computer hardware. The compiler includes all the software involved in translating a source code, such as preprocessor, assemblers and linkers.

\section{TEST CASE STUDY: PARALLEL H.263 VIDEO COMPRESSION}

\subsection{H.263 Video Compression}

The H.263 video compression standard (ITU-T, 1996) was defined by the Telecommunication Standardization Sector of the International Telecommunication Union (ITU-T) for use in a range of video applications over wireless and public switched telephone networks (PSTNs). The applications include video conferencing, video telephony, security surveillance, video streaming, and so on. H.263 is a block based hybrid code. Each picture is 


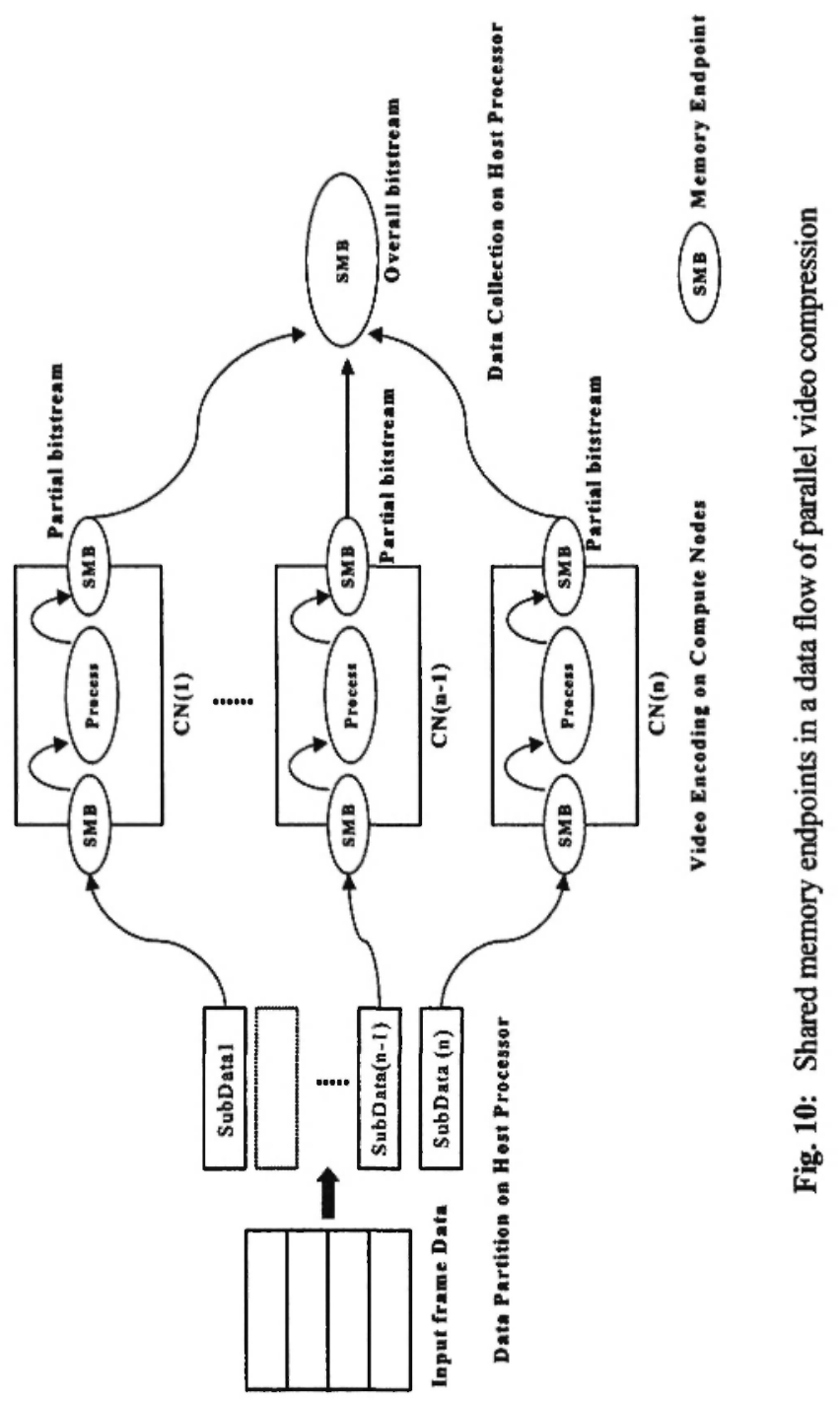


partitioned into Macro-Blocks (MB), rectangles of fixed size containing luminance and chrominance information for this rectangle. It employs motion compensated prediction followed by DCT and quantization of the error signal. Figure 11 shows a block diagram of a H.263 encoder.

The incoming pictures are divided into macroblocks. For each macroblock, motion estimation and prediction are computed based on the reconstructed previous picture. The result is a motion vector at the encoder and the predicted macroblock at the decoder. Depending on how well the predicted macroblock matches the original block, either INTRA or INTER mode is used for encoding. In INTER mode, which is used to encode most images, the difference between the block to code and the predicted macroblock is computed. This prediction error is DCT transformed and quantized using a variable quantization parameter. The resulting quantized coefficient ts is entropy coded and transmitted together with the entropy encoded motion vectors and other parameters. For reconstructing the predicted image at the encoder, the quantized and DCT-encoded prediction errors and the motion vectors are decoded at the encoder.

\subsection{Analysis of the Sequential H.263 Encoder}

The sequential H.263 encoder used is the University of British Columbia's (UBC) H.263 coder version 3.0 based on Test Model Number 5 (TMN5).

The first step before designing the parallel H.263 algorithm is to analyze the sequential H.263 algorithm, identifying different stages, including $\mathrm{I} / \mathrm{O}$ functions, setting control parameters, and encoding functions. Analysis of the execution profile of the H.263 encoder using Microsoft Visual $\mathrm{C}++6.0$ profiler on test sequences shows that most of the execution time is spent on principal encoding functions, such as motion estimation. The $\mathrm{I} / \mathrm{O}$ functions such as reading frames and saving bitstream are only small parts of the overall execution time.

The second step is data dependencies analysis. Data dependencies are the key to parallelization. In the field of video processing, another two types of data dependencies must be taken into account-the data dependency between frames and the data dependency between adjoining macroblocks. 


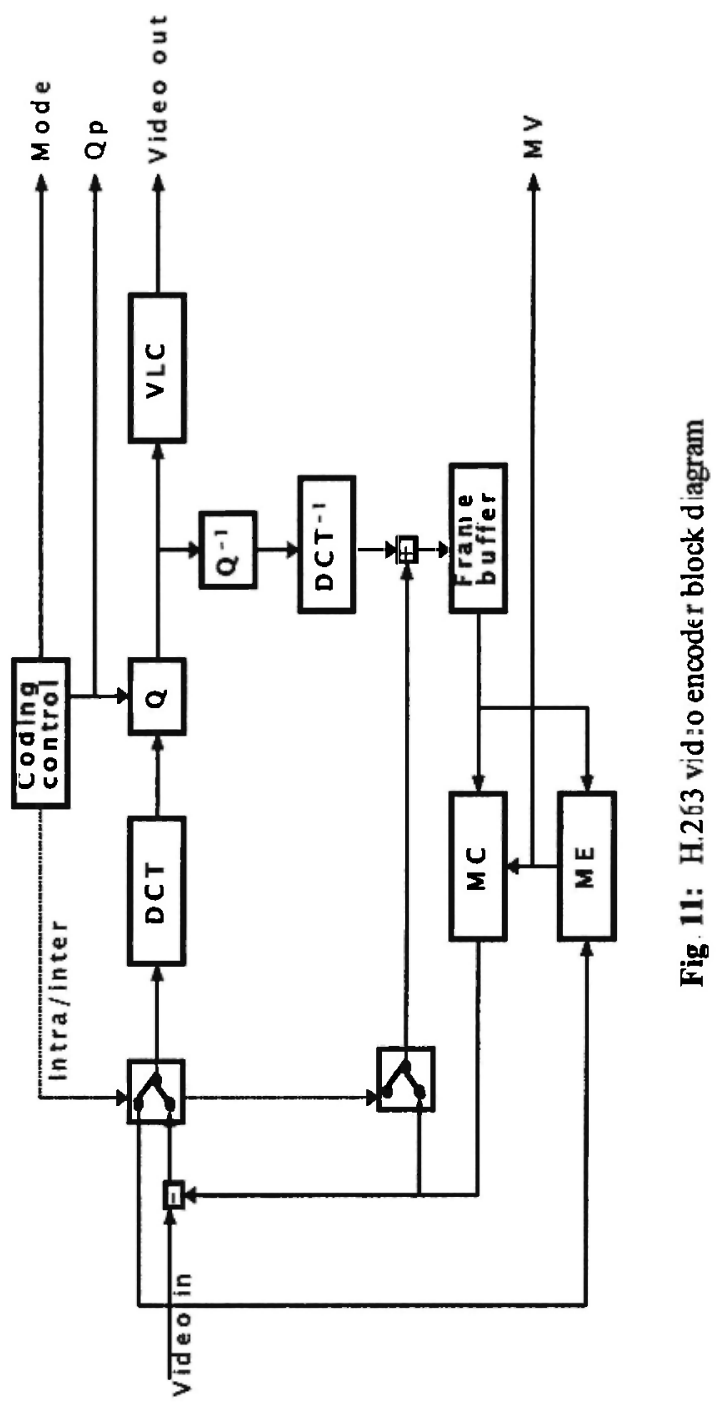


In the H.263 encoder, when using INTRA mode coding, all the pixels required for processing the DCT lie within the GOB that is being computed (Bouville et al., 1996). Hence, INTRA mode coding has no data dependency. When using INTER mode coding, the fully reconstructed frame must be ready before coding a new frame. Additionally, the motion vector components of the macroblocks are differentially coded by using a spatial neighborhood of the three motion vectors already computed. These three motion vectors are candidate predictors for the differential coding (Ghanbari, 1999).

\subsection{Implementation of H.263 Encoder on a RACE Mercury Multicomputer}

In recent years, embedded multicomputer systems with RACEway technology (Computer Systems Inc., 1994) have been used widely in defense, in medical, and in commercial signal and image processing applications, such as the airborne space-time adaptive processing (STAP) radar, high-end MRI systems, and signal intelligence processors.

4.3.1 Mercury RACE multicomputer systems. Mercury RACE multicomputer systems (Mercury Computer Systems Inc., 2005) are high-performance message-passing multicomputer systems that are used for embedded applications. These mulitcomputers provide a foundation for developing parallel systems and offer a set of building blocks that provide upward scalability. Currently, RACE multicomputers mainly use PowerPC microprocessors. Several generations of the PowerPC microprocessor have been used to build RACE multicomputer systems. The fourth generation PowerPC 7400 microprocessor (commonly known as the "G4") (Atmel, 2004) offers up to a four orders of magnitude increase over the PowerPC 750 microprocessor (G3) (Mercury Computer Systems Inc., 2000). A G5-processor-based RACE multicomputer system, XR9 system (Mercury Computer Systems Inc., 2004) has become available. XR9 systems deliver the new IBM PowerPC 8970 FX processor and are much more powerful than G3/G4-based multicomputer systems. The RACE multicomputer system grows in step with new processor generations, thus offering the flexibility required to meet growing computing power demands.

The platform used in this study is the Mercury RACE multicomputer as shown in Fig. 12. This multicomputer consists of a PC host (Pentium III 
$500 \mathrm{MHz}$ with $128 \mathrm{MB}$ RAM), accelerated with VantageRT PowerPC PCI Base Modules as shown in Fig. 13.

Fig. 12: Mercury VantageRT system

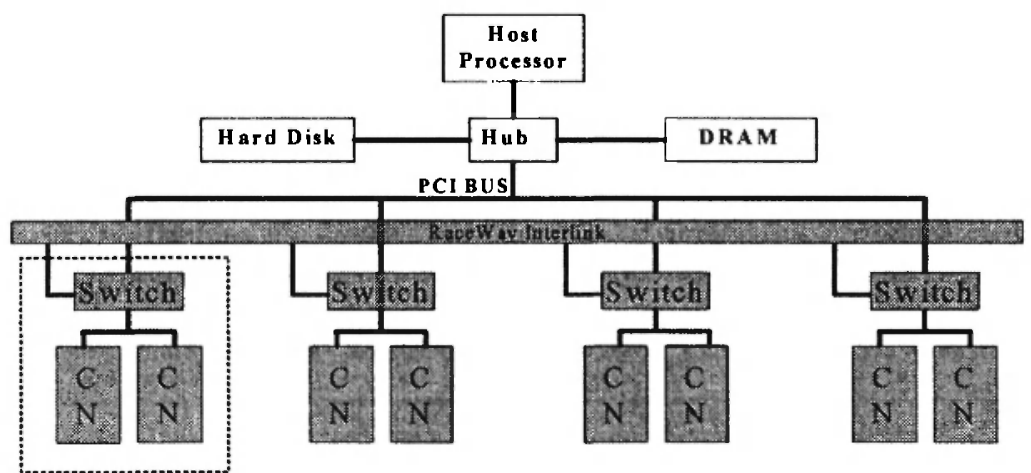

PCI VantageR T

Base Modulc

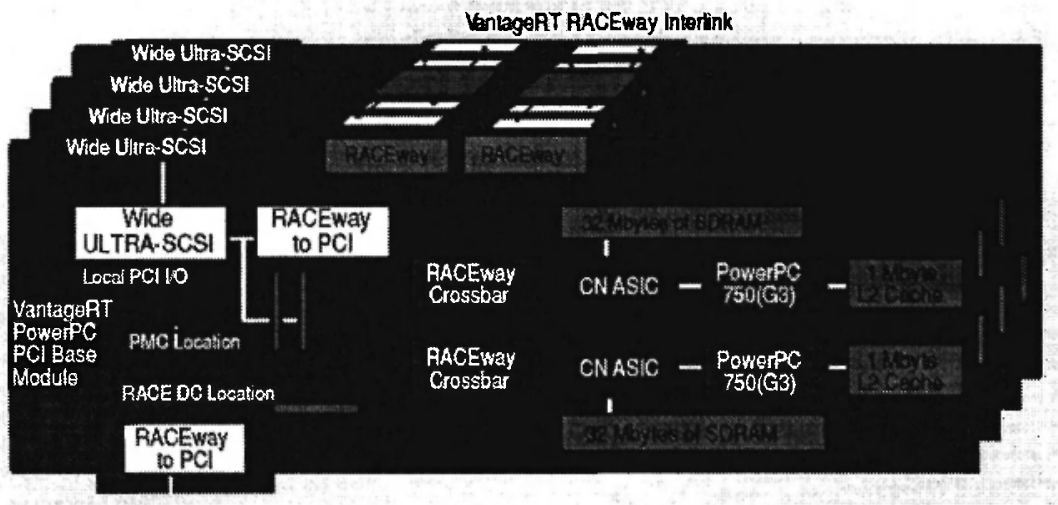

Fig. 13: PCI VantageRT base module architecture 
This standard PCI card includes two PowerPC 750 CNs and a RACEway switched-fabric interconnect that is fully accessible to the host bus. Each $\mathrm{CN}$ includes a PowerPC 750 RISC processor (CPU frequency $292 \mathrm{MHz}$, commonly known as the "G3") (Mercury Computer Systems Inc. 1999a), 1MB backside L2 cache, and $32 \mathrm{MB}$ SDRAM, managed by a Mercury ASIC that interfaces to the RACEway switched fabric. The RACEway switched fabric provides high-bandwidth data paths between processors on the base module, optional mezzanine card locations, and additional VantageRT boards. Two RACEway ports off the board provide a $320 \mathrm{MB} / \mathrm{s}$ communication path that scales up to $1 \mathrm{~GB} / \mathrm{s}$ between multiple VantageRT boards in the same host chassis. This workstation is performance-scalable and can be configured with up to 16 base modules, for a maximum capacity of 32 processors.

The software foundation of the RACE multicomputer is the MC/OSTM runtime environment (Mercury Computer Systems Inc. 1999b). The MC/ OSTM multicomputer operating environment is a collection of runtime facilities, libraries, and development tools targeted at multiprocessor application development on all Mercury multicomputer systems. The runtime and development environments contain APIs and tools that address not only the algorithms that run on each processor but also the communication and coordination of processes distributed across multiple processors.

4.3.2 Mapping strategy for parallel H.263 encoder. For data parallelism, two approaches can be used to partition the video data-spatial parallelism and temporal parallelism (Ahmad et al., 2002). Spatial parallelism partitions the video data into groups of slices or groups of macroblocks, and temporal parallelism partitions the video data into groups of pictures (GOPs). Spatial parallelism is considered more suitable for real-time video compression because it introduces less coding delay than temporal parallelism. Thus, spatial parallelism is used in this implementation. The input frames are read frame-by-frame into a memory buffer at the host processor. One important question is how to schedule each frame data to processors with minimum load unbalancing and scheduling overhead.

To minimize the load unbalance, each $\mathrm{CN}$ must process the frame data as equally as possible. Yet, repartitioning the frame data into subsections on each frame on the host processor introduces overhead, causing a considerable amount of coding delay. To avoid this overhead, the whole frame data are 
scheduled to each processor. Then each processor accesses a different section of the frame data. Due to frame dependencies, processors may have to refer to a previous frame and its encoding results. This approach allows overlapping of frame data on each $\mathrm{CN}$, thus saving communication time.

The frame data are accessed by each $\mathrm{CN}$ horizontally rather than vertically because the image is read and saved horizontally, left to right, row by row. Accessing the video data in storage order also reduces the overhead incurred by accessing the data structures. The boundaries of each section (i.e. startline and endline) are scheduled statically for each $\mathrm{CN}$ as part of coding parameters. With the data partition at the Group Of Block (GOB) level, the number of GOBs per picture is 9 for QCIF and 18 for CIF image, so the maximum number of CNs that can be used is 9 for QCIF image and 18 for CIF image.

For implementations of encoding QCIF video on $3 / 9 \mathrm{CNs}$ or CIF video on $2 / 3 / 6 / 9 / 18 \mathrm{CNs}$, each $\mathrm{CN}$ is assigned with equal GOBs. In these cases, the parallel implementation achieves load balance. For other implementations, equally dividing the GOBs into equal parts is impossible, so the CNs assigned with a less number of GOBs will finish the processing quicker. The latter have to wait for the slowest $\mathrm{CN}$ to finish the processing before carrying on encoding the next frame. As shown in Fig. 14, the frame data are transferred to each compute node's DRAM individually.

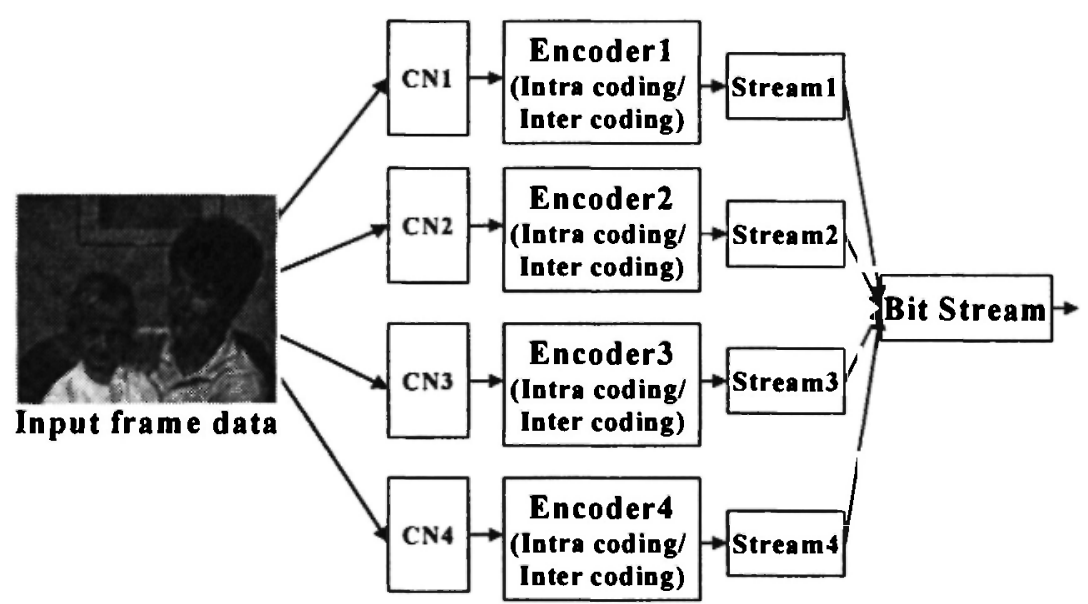

Fig. 14: H.263 parallel implementation scheme 
4.3.3 Synchronization. Synchronization is carried out by sending and receiving commands among the processors using the linear pipeline communication topology shown in Fig. 2. The commands issued by the host processor are passed to the first node and then propagated through the pipeline until reaching the last node. Each node responds to the commands received from the previous node and carries out the computation. According to the data dependency analysis, when coding a new frame in INTER mode coding, the fully reconstructed frame is generated on the host processor and passed to each $\mathrm{CN}$ before coding.

As the motion vector components of the macroblocks are differentially coded by using a spatial neighborhood of three motion vectors already computed (Ghanbari, 1999), motion estimation is performed on each region plus the row of macroblocks immediately above the given region to perform motion prediction in parallel on each horizontal region of an image (Cosmas et al., 1998). As the whole frame data are on each $\mathrm{CN}$, exchanging boundary data among $\mathrm{CNs}$ is not necessary.

When it finishes encoding its own frame data, the first node passes to the next node a flag, indicating that it has finished processing. After a node has finished processing its own data, it waits until the flag has arrived from the previous node. Then, it passes the flag to the next node and so on until the flag reaches the last node. The last node in the pipeline sends an acknowledgement signal and information of each computed partial result to the host processor, indicating that all the nodes have finished processing. Next, the host issues a command to tell each node to send its coded partial bitstream and reconstructed partial image back to the host. Finally, the host collates all the partial bitstreams and adds them to the global $\mathrm{H} .263$ bitstream in a buffer or a bitstream file. In addition, it merges the reconstructed partial image to form a fully reconstructed image. The fully reconstructed image is sent to each $\mathrm{CN}$ for the encoding of next frame. Then the encoding for the next frame begins.

4.3.4 Data transfer. Both the Memory Copy and DMA transfer approaches are used in this implementation. In the Memory Copy approach, the data move very fast between processors if the transfer of data between processors is smaller than 100 Kbytes. When the data size exceeds $200 \mathrm{Kbytes}$, then the 
bandwidth begins to stay at around $13 \mathrm{Mbytes} / \mathrm{sec}$. For the DMA transfer approach, the bandwidth of sending/receiving data between two processors

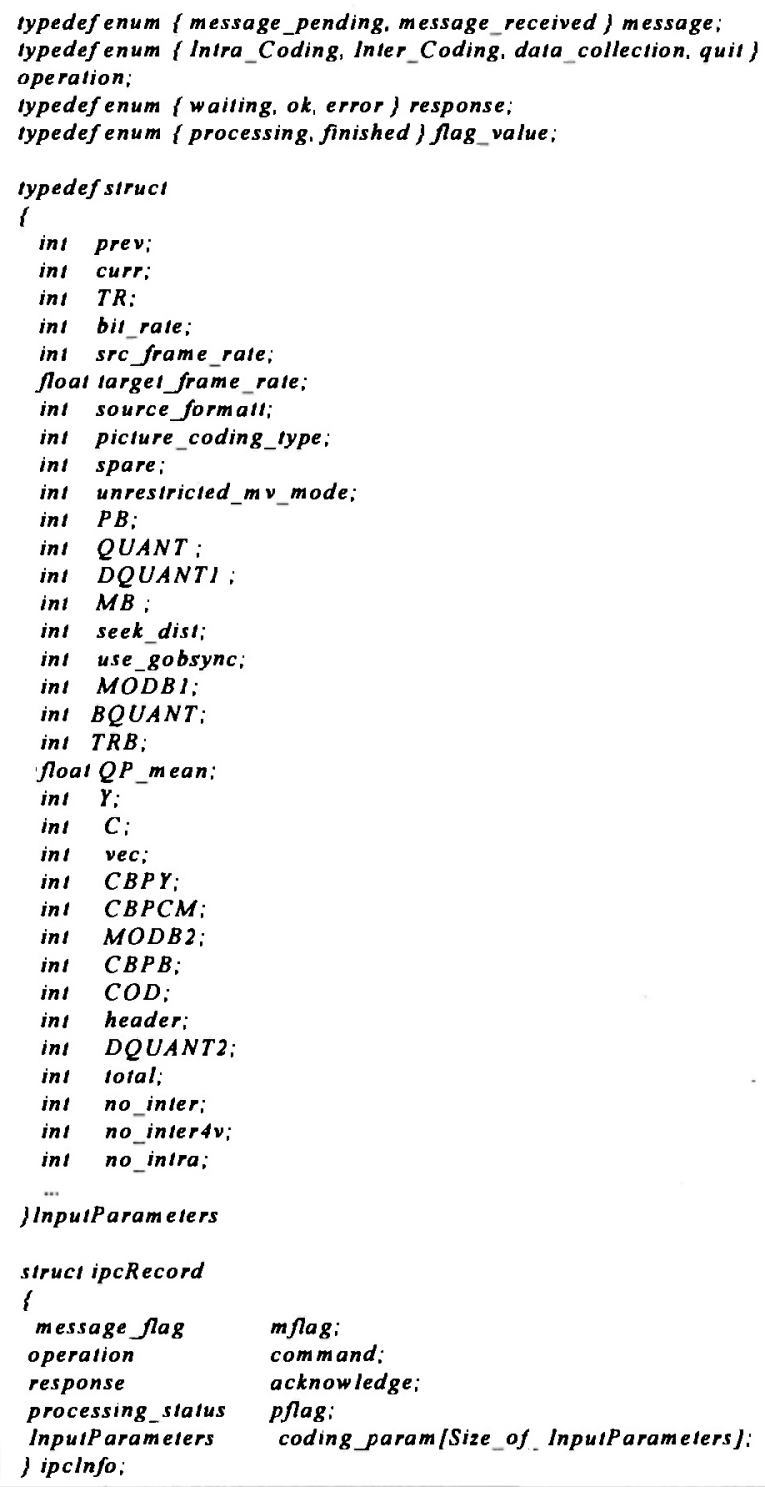

Fig. 15: Message structure for parallel H.263 encoder 
with a DMA transfer is about $100 \mathrm{MB} / \mathrm{s}$. Thus, the first approach is used for transferring message and small size of data ( $<100$ Kbytes), and the later approach is used for transferring a larger size of data (>100 Kbytes).

4.3.5 Writing the non-accelerated and the accelerated code. Based on the methodology proposed in Sec. 2, the non-accelerated and the accelerated codes for parallel H.263 encoder are designed. A message structure is designed as shown in Fig. 15. Figure 16 shows the Pipeline Pseudo Codes for the host processor and all the Compute Nodes.

4.3.6 Compiling. The non-accelerated code scheduled on host processor is compiled using the Microsoft Visual $\mathrm{C}+6.0$ compiler. The Mercury compiler ccmc then translates the accelerated codes into native codes to execute on the compute codes. For the H.263 encoder, the computation procedures are in the separate $\mathrm{C}$ programs, and then the compiler needs to compile these $\mathrm{C}$ programs and link their object files into an executable program. An example of compiling an accelerated code for $\mathrm{CN}(1)$ is given in Fig. 17. ppc1.ppc_Ie is the generated executable program.

4.3.7 Implementation results. YUV 4:2:0 sequences are used as input for the encoding process. We have used three test video sequences of QCIF and CIF resolutions (Fig. 18). These video sequences cover the slow motion and fast motion types. Mother and Daughter is a low complexity head and shoulder sequence. Foreman has a medium complexity and Calendar and Mobile is a high complexity sequence, with a lot of movement including rotation. As the complexities of $\mathrm{H} .263$ coding in four optional modes do not increase a lot compared with the complexity of the baseline mode (i.e. all the optional modes are turned off), thus only the baseline mode coding is considered in this implementation. For motion estimation algorithm, the full search scheme is used in this implementation. For easy comparison, in this parallel H.263 implementation, the following coding parameters are set as default and stay the same for all the tests:

Coding mode = baseline;

Reference frame rate $=30$;

Search seek distance $=15$; (full search)

Intra quantization parameter $=10$;

Inter quantization parameter $=10$;

Number of skipped frames between encoded frames $=0$ (no skipping frames) 

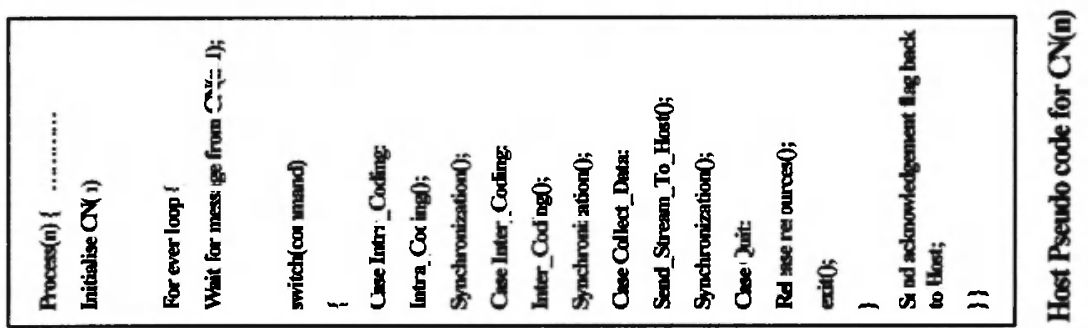

己ั
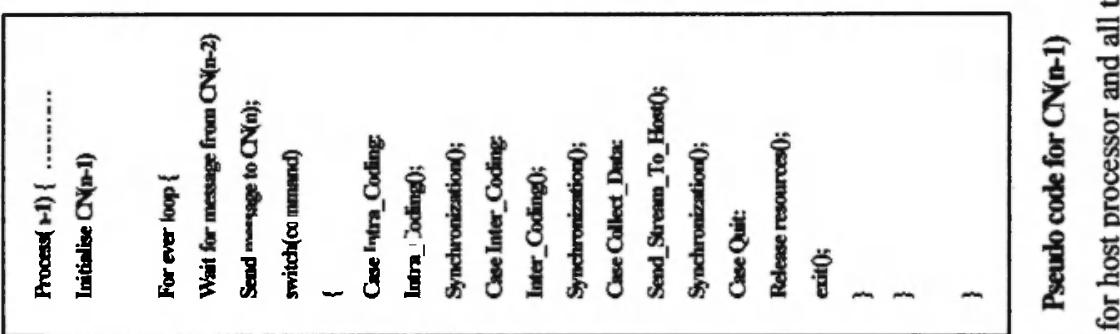

$\vdots$

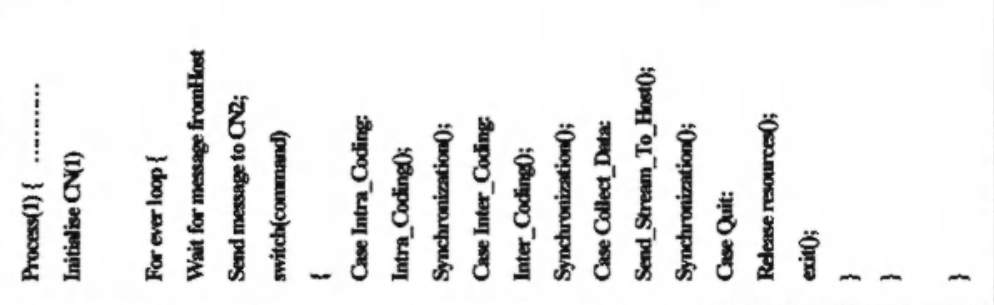

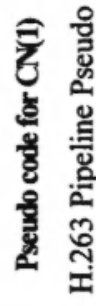

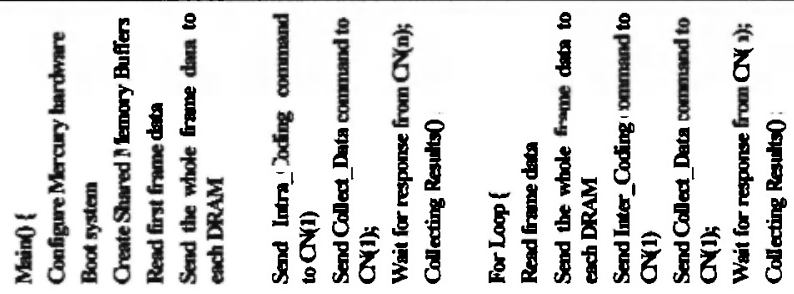

음 
ccmc -t ppc_le - g -c -o putbits oppc_le putbits c

ccmc -t ppc_le - $\mathrm{g}-\mathrm{c}-\mathrm{o}$ det. oppc_le det c

ccme -t ppc_le-g-c-o io.oppc_le io.c

ccmc -t ppc_le -g -c -o sac oppc_le sac.c

ccmc - $t$ ppc le - $-\mathrm{g}-\mathrm{c}-\mathrm{o}$ quant oppc le quant.

ccmc -t ppc_le - $\mathrm{g}-\mathrm{c}-\mathrm{o}$ mot_est.oppc_le mot_est.c

ccmc -t ppc le -g -c -o pred.oppc_le pred.c

ccmc-t ppc_le - B -c -o countbit oppc_le countbit c

ccmc-t ppc_le -g - - -0 putvlc.oppc_le putvlc.c

$c c m c-t p p c \_l e-g-c-o$ quant oppc_le quant.c

ccme -t ppc_le - $\mathrm{g}-\mathrm{c}$-o snr oppc_le snr.c

ccmc -t ppc_le - $\mathrm{g}-\mathrm{c}-\mathrm{o}$ coder oppc_le coder $\mathrm{c}$

ccmc -t ppc_le - $\mathrm{g}-\mathrm{c}-\mathrm{o}$ ratectrloppc_le ratectrl c

ccme-1 ppc_le -g-c-o ppcl oppc_le ppcl c

ccmc -t ppc_le -m ppcl map -g -o ppc1.ppc_le ppcl oppc_le coder.oppc_le putbits.oppc_le

putvlc oppc_le det.oppc_le 10.oppc_le pred.oppc_le snr.oppc_le countbit.oppc_le mot_est.oppc_le

ratectrloppc_le sac.oppc_le quant oppc_le

Fig. 17: Makefile for compiling an accelerated code

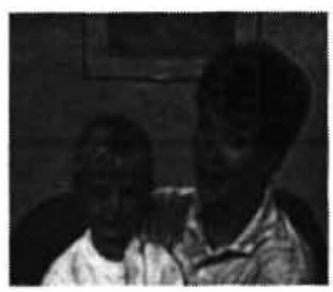

Mother and Doughter

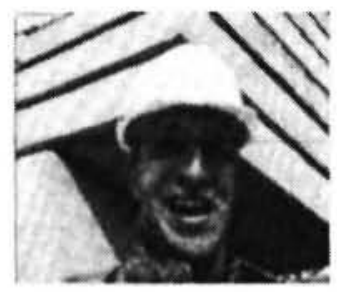

Foreman

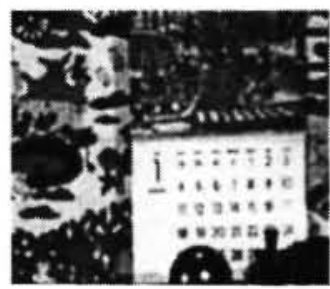

Calendar and Mobile

Fig. 18: Three test video sequences with different motion types

The overall execution time is measured on the PC host processor, including data reading and saving, command sending and receiving, data transferring, and the processing time on the multicomputer. To reduce the influence of disk caching on the execution time, the same sequence is encoded three times in a row. As the purpose is to measure the performance of the encoding process, not any $\mathrm{I} / \mathrm{O}$ operations, only the last execution is taken into account. The proportional time usage of $\mathrm{I} / \mathrm{O}$ operations is very small in the last execution, because the disk cache is filled during the two previous executions. The results of execution times, speedups as well as frame encoding rates using different number of processors are shown below. 
4.3.7.1 $Q C I F$, video encoding results. Tables 1,2 , and 3 show the results of encoding QCIF test sequences with different complexities.

TABLE 1

Results of encoding low complexity QCIF video

(Mother and Daughter (176x144), 60 frames)

\begin{tabular}{|l|l|l|l|l|l|}
\hline $\begin{array}{l}\text { No. of } \\
\text { Processors }\end{array}$ & $\begin{array}{l}\text { Execution } \\
\text { time } \\
\text { (seconds) }\end{array}$ & $\begin{array}{l}\text { Actual } \\
\text { Speedup }\end{array}$ & $\begin{array}{l}\text { Ideal } \\
\text { Speedup }\end{array}$ & $\begin{array}{l}\text { Encoding Rate } \\
\text { (frames/second) }\end{array}$ & $\begin{array}{l}\text { Parallel } \\
\text { Efficiency }\end{array}$ \\
\hline 1 & 3.244 & - & - & 18.51 & - \\
\hline 2 & 2.534 & 1.28 & 1.8 & 23.67 & $61.4 \%$ \\
\hline 3 & 1.852 & 1.75 & 3 & 32.40 & $58.3 \%$ \\
\hline 4 & 1.582 & 2.05 & 3 & 37.93 & $51.25 \%$ \\
\hline
\end{tabular}

TABLE 2

Results of encoding medium complexity QCIF video

(Foreman (176x144), 60 frames)

\begin{tabular}{|l|l|l|l|l|l|}
\hline $\begin{array}{c}\text { No. of } \\
\text { Processors }\end{array}$ & $\begin{array}{c}\text { Execution } \\
\text { time } \\
\text { (seconds) }\end{array}$ & $\begin{array}{c}\text { Actual } \\
\text { Speedup }\end{array}$ & $\begin{array}{c}\text { Ideal } \\
\text { Speedup }\end{array}$ & $\begin{array}{c}\text { Encoding Rate } \\
\text { (frames/second) }\end{array}$ & $\begin{array}{c}\text { Parallel } \\
\text { Efficiency }\end{array}$ \\
\hline 1 & 4.396 & - & - & 13.64 & - \\
\hline 2 & 2.955 & 1.49 & 1.8 & 20.30 & $74.5 \%$ \\
\hline 3 & 2.303 & 1.91 & 3 & 26.05 & $63.7 \%$ \\
\hline 4 & 1.962 & 2.24 & 3 & 30.58 & $56 \%$ \\
\hline
\end{tabular}

TABLE 3

Results of encoding high complexity QCIF video

(Calendar \& Mobile (176x 144), 60 frames)

\begin{tabular}{|l|l|l|l|l|l|}
\hline $\begin{array}{c}\text { No. of } \\
\text { Processors }\end{array}$ & $\begin{array}{c}\text { Execution } \\
\text { time } \\
\text { (seconds) }\end{array}$ & $\begin{array}{c}\text { Actual } \\
\text { Speedup }\end{array}$ & $\begin{array}{c}\text { Ideal } \\
\text { Speedup }\end{array}$ & $\begin{array}{c}\text { Encoding Rate } \\
\text { (frames/second) }\end{array}$ & $\begin{array}{c}\text { Parallel } \\
\text { Efficiency }\end{array}$ \\
\hline 1 & 5.287 & - & - & 11.35 & - \\
\hline 2 & 3.385 & 1.56 & 1.8 & 17.73 & $78 \%$ \\
\hline 3 & 2.413 & 2.19 & 3 & 24.87 & $73 \%$ \\
\hline 4 & 2.262 & 2.34 & 3 & 26.53 & $58.5 \%$ \\
\hline
\end{tabular}


4.3.7.2 CIF, video encoding results. The following Tables 4, 5 , and 6 show the results of encoding CIF test sequences with different complexities.

TABLE 4

Results of encoding low complexity CIF video

(Mother and Daughter (352×288), 60 frames)

\begin{tabular}{|c|c|c|c|c|c|}
\hline $\begin{array}{c}\text { No. of } \\
\text { Processors }\end{array}$ & $\begin{array}{c}\text { Execution } \\
\text { time } \\
\text { (seconds) }\end{array}$ & $\begin{array}{c}\text { Actual } \\
\text { Speedup }\end{array}$ & $\begin{array}{c}\text { Ideal } \\
\text { Speedup }\end{array}$ & $\begin{array}{c}\text { Encoding Rate } \\
\text { (frames/second) }\end{array}$ & $\begin{array}{c}\text { Parallel } \\
\text { Efficiency }\end{array}$ \\
\hline 1 & 13.560 & - & - & 4.42 & - \\
\hline 2 & 8.482 & 1.60 & 2 & 7.07 & $80 \%$ \\
\hline 3 & 6.218 & 2.18 & 3 & 9.65 & $72.7 \%$ \\
\hline 4 & 5.348 & 2.54 & 3.6 & 11.22 & $63.5 \%$ \\
\hline
\end{tabular}

TABLE 5

Results of encoding medium complexity CIF video

(Foreman $(352 \times 288), 60$ frames)

\begin{tabular}{|c|c|c|c|c|c|}
\hline $\begin{array}{c}\text { No. of } \\
\text { Processors }\end{array}$ & $\begin{array}{c}\text { Execution } \\
\text { time } \\
\text { (seconds) }\end{array}$ & $\begin{array}{c}\text { Actual } \\
\text { Speedup }\end{array}$ & $\begin{array}{c}\text { Ideal } \\
\text { Speedup }\end{array}$ & $\begin{array}{c}\text { Encoding Rate } \\
\text { (frames/second) }\end{array}$ & $\begin{array}{c}\text { Parallel } \\
\text { Efficiency }\end{array}$ \\
\hline 1 & 18.166 & - & - & 3.30 & - \\
\hline 2 & 10.766 & 1.69 & 2 & 5.57 & $84.5 \%$ \\
\hline 3 & 7.651 & 2.37 & 3 & 7.84 & $79 \%$ \\
\hline 4 & 6.799 & 2.67 & 3.6 & 8.82 & $66.8 \%$ \\
\hline
\end{tabular}

TABLE 6

Results of encoding high complexity CIF video

(Calendar \& Mobile (352×288), 60 frames)

\begin{tabular}{|c|c|c|c|c|c|}
\hline $\begin{array}{c}\text { No. of } \\
\text { Processors }\end{array}$ & $\begin{array}{c}\text { Execution } \\
\text { time } \\
\text { (seconds) }\end{array}$ & $\begin{array}{c}\text { Actual } \\
\text { Speedup }\end{array}$ & $\begin{array}{c}\text { Ideal } \\
\text { Speedup }\end{array}$ & $\begin{array}{c}\text { Encoding Rate } \\
\text { (frames/second) }\end{array}$ & $\begin{array}{c}\text { Parallel } \\
\text { Efficiency }\end{array}$ \\
\hline 1 & 23.443 & - & - & 2.56 & - \\
\hline 2 & 13.109 & 1.79 & 2 & 4.56 & $89.5 \%$ \\
\hline 3 & 8.752 & 2.68 & 3 & 6.86 & $89.3 \%$ \\
\hline 4 & 8.582 & 2.73 & 3.6 & 6.99 & $68.3 \%$ \\
\hline
\end{tabular}


From the above results, we can see that the performance/complexity of the H.263 encoder can be different because of the variety of motion in the scene. The complexity of the H.263 encoder becomes high many movements are in the scene because more computations are required. Additionally, we can see that as the number of processors increases, the parallel efficiency decreases accordingly because the communication overhead increases.

Based on the above results, Figs. 19 and 20 illustrate how speedups and encoding frame rates are improved accordingly when the number of processors used is increased.

In Fig. 19, we can see that as the number of processors increases, the speedup increases accordingly, but not as great as the ideal speedup due to the data movement and synchronization overheads. The speedups of CIF image encoding using additional processors are estimated and shown by dashed lines. In those cases when load balance is achieved (the frame data are equally processed), the parallel efficiencies are higher than those when load balance is not achieved.

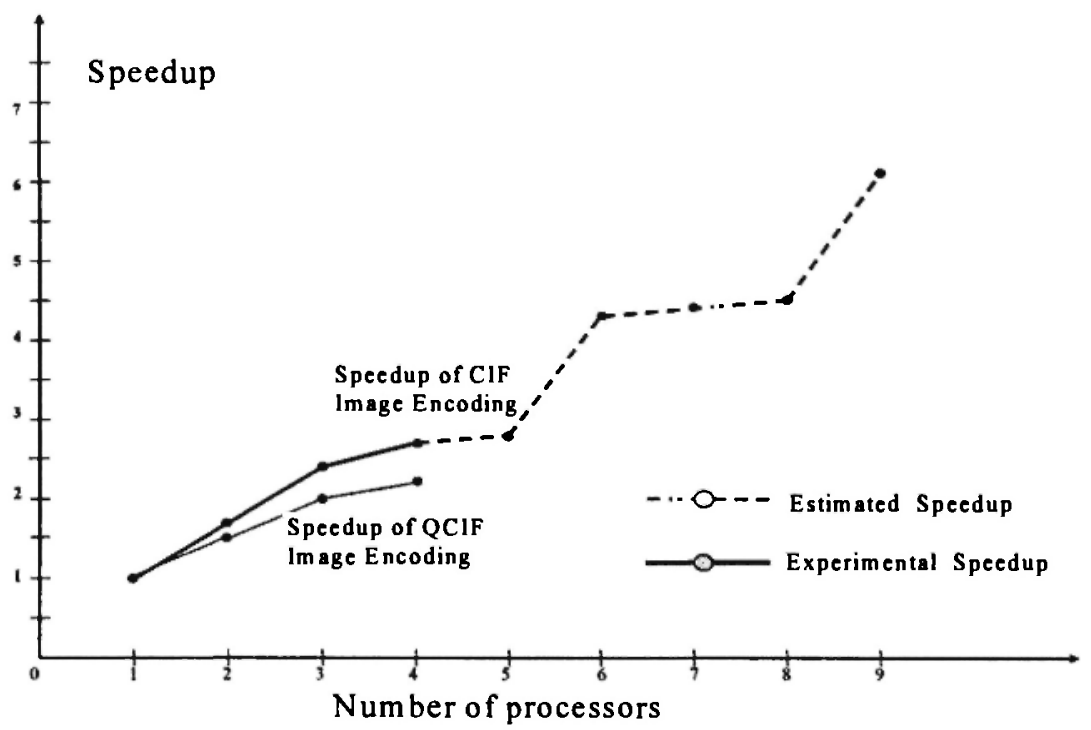

Fig. 19: Speedup versus number of processors 
From Fig. 20, we can see that real-time performance has been achieved when encoding QCIF images with three processors. With four processors, the encoding rate is faster than real-time performance.

For CIF image encoding, the performance of 11.22 frames per second has been achieved by using four G3 processors. Similarly, dashed lines indicate the encoding rates of using additional processors. It is estimated that with nine $\mathrm{G} 3$ processors, real-time CIF encoding can be achieved.

With the newer generation processor-PowerPC 7410 using the Alti$\mathrm{Vec}^{\mathrm{TM}}$ technology (Atmel, 2004)-the performance can be approximately four times faster than PowerPC 750 processor (Mercury Computer Systems Inc., 2000). Based on this assumption, the encoding trame rates of CIF image encoding using G4 processors are estimated and shown by dashed lines. To achieve real-time QCIF encoding performance, only one G4 processor is required. With two G4 processors, real-time CIF encoding can be achieved.

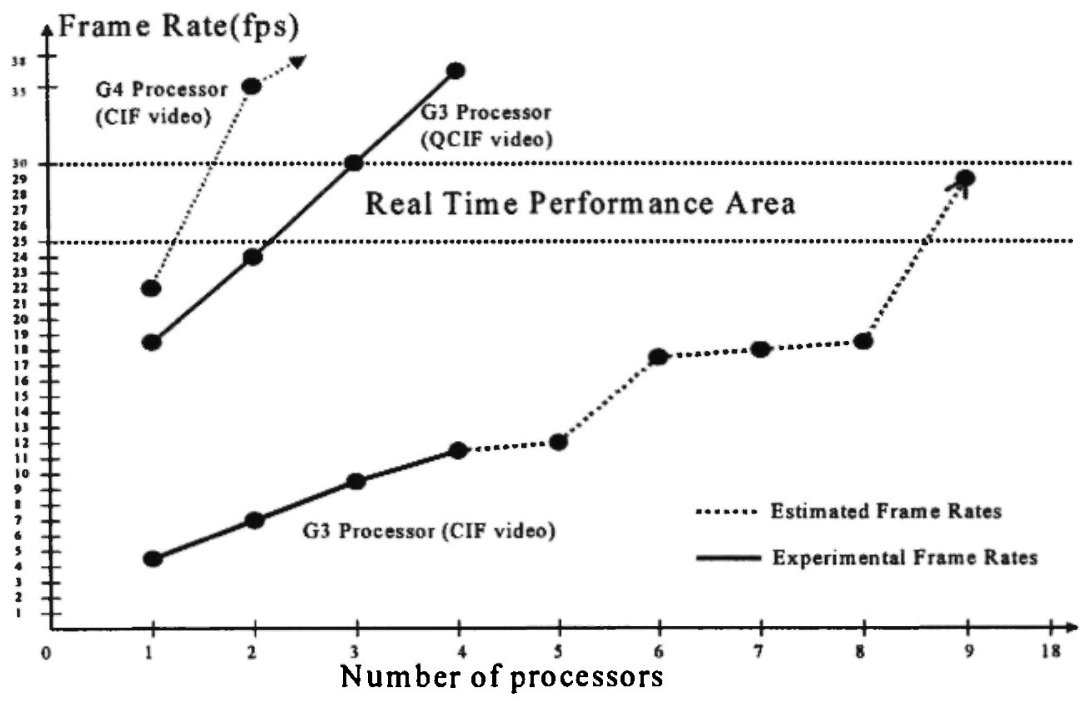

Fig. 20: Frame rate versus number of processors 


\section{CONCLUSIONS}

The generic parallel processing framework proposed in this paper allows the easy prototyping of new parallel algorithms. It ensures the successful synchronization between execution of non-accelerated code on the host processor and accelerated code on the multiprocessors. We demonstrated how the framework can be used in real-time software video compression. The implementation of a parallel H.263 encoder using the framework showed that a software approach with high-performance embedded multicomputer systems can meet the high demands of real-time video compression. The developed parallel processing framework can be applied to parallelizing certain other video codecs as well, such as MPEG-2, MPEG-4 and H.264. Finally, this framework provides a good software implementation solution as an alternative to hardware implementation to achieve high-quality real-time video encoding for converged broadcast and mobile services. Flexibility, expandability, and interactivity are the three compelling reasons for the transition from a hardware solution to this software solution.

\section{ACKNOWLEDGMENTS}

The authors gratefully acknowledge the support for this work funded by the European Union under the Information Society Technologies program as the project CISMUNDUS (Convergence of IP-based Services for Mobile Users and Networks in DVB-T and UMTS Systems): www.brunel.ac.uk/ project/cismundus.

\section{REFERENCES}

Ahmad, I., He, Y. and Liou, M.L. 2002. Video compression with parallel processing, Parallel Computing, 28, 1039-1078.

Akramullah, S.M., Ahmad, I. and Liou, M.L. 1995. A data-parallel approach for real-time MPEG-2 video encoding, Journal of Parallel and Distributed Computing, 30, 129-146. 
Akramullah, S.M., Ahmad, I. and Liou, M.L. 1997. A software based H.263 video encoder using network of workstations, Proceedings of SPIE, 3166.

Atmel. 2004. PowerPC 7410 RISC Microprocessor product specification, www.atmel.com/dyn/resources/prod_documents/doc2141. pdf.

Barbosa, D.M. et al. 1999. Parallelizing MPEG video encoding using multiprocessors, Proceedings of XII Brazilian Symposium on Computer Graphics and Image Processing, 215-222.

Barbosa, D., Kitajima, J.P. and Meira, W. Jr. 1999. Real-time MPEG encoding in shared-memory multiprocessors, Proceedings of the $2^{\text {nd }}$ International Conference-Parallel Computing Systems 1999, Ensenada, Baja California, Mexico.

Bouville, C., Houlier, P., Doubois, J.L., Marchal, I., Thebault, B. and Klefstad, M. 1996. A flexible MPEG real time video codec, ICIP-96, 3, 829-832.

Cosmas, J.P., Paker, Y. and Pearmain, A.J. 1998. Parallel H.263 video encoder in normal coding mode, IEE Electronics Letters, 34, 2109-2110.

Einstein T.H. 1997. Mercury Computer systems' modular heterogeneous RACE multicomputer, in: Proceedings of the Sixth Heterogeneous Computing Workshop (HCW '97), Geneva, Switzerland, IEEE Computer Society, 60-71.

Envivio. 2004. Envivio's 4 Caster encoder, www.envivio. com.

Fernández, J.C. and Malumbres, M.P. 2002. A parallel implementation of H.26L Video Encoder, Euro-Par 2002, 830-833.

Ghanbari, M. 1999. Video coding: an introduction to standard codecs, 42, IEE Telecommunications Series, IEE Publishing.

Harmonic. 2004. Harmonic the DiviCom MV encoders, www.harmonic.com.

Hatcher, P.J., Quinn, M.J. and Lapadula, A.J. 1991. Data-parallel programming on MIMD computers, IEEE Transactions on Parallel and Distributed Systems, 2, 377-383.

He, Y., Ahmad, I. and Liou, M.L. 1999. Real-time interactive MPEG-4 system encoder using a cluster of workstations, IEEE Transactions on Multimedia, 1.

ITU-T [Telecommunication Standardisation sector of International Communication Union]. 1990. Recommendation H.261, video codec for audiovisual services at $\mathrm{p} \times 64 \mathrm{kbit} / \mathrm{s}, I T U-T$ Recommendation H.261.

ITU-T [Telecommunication Standardisation sector of International Communication Union]. 1996. Video coding for low bit-rate communication, ITU. T Recommendation H.263.

ITU-T [Telecommunication Standardisation sector of International Communication Union]. 2003, Advanced video coding for generic audiovisual services, ITU-T Recommendation H.264.

Kolinummi, P., P. Sarkijarvi, J. Hamalainen, T. Saarinen, J. 2000. Scalable implementation of $\mathrm{H} .263$ video encoder on a parallel DSP system, IEEE International Symposium on Circuits and Systems, 1, 551-554. 
Kuszmaul, B.C. 1995. The RACE network architecture, Proceedings of $9^{\text {th }}$ International Parallel Processing Symoposium, Santa Barbara, California, April 25-28, Washington, DC, USA, IEEE Computer Society Press, 508-513.

Leung, K.K., Yung, N.H.C. and Cheung, P.Y.S. 2000. Parallelization methodology for video coding - an implementation on TMS320C80, IEEE Transaction on Circuits and Systems for Video Technology, 10, 14131425.

Li, E.Q. and Chen, Y. 2004. Implementation of H.264 encoder on generalpurpose processors with hyper-threading technology, Proceedings of SPIE Visual Communications and Image Processing, 384-395.

Lin, W., Goh, K.H., Tye, B.J. and Powell, G.A. 1997. Real time H.263 video codec using parallel DSP, IEEE International Conference Image Processing, 2, 586-589.

Mercury Computer System Inc. 1994. The RACEway specification, 'ANSI/ VITA 5-1994.

Mercury Computer System Inc. 1999a. RACE series VantageRT PowerPC 750 PCI base modules, www.mc.com/Data_sheets/1999/vantagert.pdf.

Mercury Computer System Inc. 1999b. MC/OS Runtime environment, www. mc.com/Data_sheets/dsmcos.pdf.

Mercury Computer System Inc. 2000. Mercury Computer Systems supercharges PCI product line with "G4" Processors and RACE++: New VantageRT 7400 computes image and signal processing algorithms four times faster than earlier products, news on 12/12/2000, www.mc.com/ press/2000_12_12_vantagert7400.html.

Mercury Computer System Inc. 2005. Mercury Computer System, www.mc. com.

Mercury Computer System, Inc. 2004. XR9 systems, www.mc.com/literature/ literature_files/XR_Systems-ds.pdf.

MPEG-1 video group. 1993. Information technology-coding of moving pictures and associated audio for digital storage media up to about $1.5 \mathrm{Mbit} / \mathrm{s}$ : Part 2-Video, ISO/IEC 11172-2, International Standard.

MPEG-2 video group. 1995. Information technology-generic coding of moving pictures and associated audio: Part 2-Video, ISO/IEC 13818-2, International Standard.

MPEG-4 video group. 1998. Generic coding of audio-visual objects: Part 2Visual, ISO/IEC JTCI/SC29/WG1I N1902, FDIS of ISO/IEC 14496-2, Atlantic City, New Jersey, USA.

Nicolas, H. and Jorda, F. 1996. Interactive optimization and massively parallel implementations of video compression algorithms, International Conference on Image Processing, 1, 229-232.

Optibase. 2004. Optibase's MPEG MovieMaker encoder, www.optibase. com

Shen, K. and Delp, E.J. 1995. $\therefore$ parallel implementation of an MPEG encoder: Faster than real-time! Proceedings of the SPIE Conference on Digital Video Compression: 'Algorithms and Technologies, 407-418. 
Tiwari, P. and Viscito, E. 1996. A parallel MPEG-2 video encoder with lookahead rate control, IEEE International Conference on Acoustics, Speech, and Signal Processing, 4, 1994-1997.

Wiegand, T., Sullivan, G. J., Bjontegaard, G. and Luthra, A. 2003. Overview of the H.264/AVC video coding standards, IEEE Transactions on Circuits and Systems for Video Technology, 560-576.

Yung, N.H.C. and Chu, K.C. 1998. Fast and parallel video encoding by workload balancing, IEEE International Conference on Systems Man and Cybernetics, 5, 4642-4647. 
